\title{
On the Independence of the Thermodynamic Limit on the Boundary Conditions in Quantum Statistical Mechanics
}

\author{
N. Angelescu and G. Nenciu \\ Institute for Atomic Physics, Bucharest
}

Received July 1, 1972

\begin{abstract}
The problem of the independence of the thermodynamic limit on the boundary conditions is considered in the framework of functional integration. For every domain and every boundary condition in a sufficiently large class a functional measure is constructed and the Feynman-Kac-like formula for the statistical operator written down. Making use of some volume-independent estimates for the Green function of the heat equation, the thermodynamic limit along convex domains for general boundary conditions is proved to exist and to be equal to that for Dirichlet conditions.
\end{abstract}

\section{§ 1. Introduction}

Essentially two methods have been used in quantum statistical mechanics for including the boundary conditions in the definition of local hamiltonians. One of them employs the connection between semibounded sesquilinear forms over a Hilbert space and semibounded selfadjoint operators $[1,2]$. The other makes use of functional integration to write Feynman-Kac formulae for the semigroup of statistical operators $\exp (-\beta H), \beta \geqq 0$. Initially devised to accomodate Dirichlet boundary conditions [3], this method was extended by Novikov [4], who considered the functional measure associated to the Wiener process in a parallelepipedic box with elastic reflecting walls and has thus been able to handle the case of Neumann boundary conditions for such domains

This paper is concerned with extending the second method for a larger class of domains and boundary conditions. In Section 2, functional measures suited for a class of boundary conditions are constructed, following the standard way [5] and using the appropriate Green function of the heat equation. Different properties of these measures, which follow from local estimates of the Green function, and the relation with the measures used by Ginibre [3] and Novikov [4] is established. In Section 3, the Feynman-Kac formula is written down and the equality of the thermodynamical limits for the whole class of boundary conditions 
is proved. The infinite volume limit is to be taken along sequences of convex domains with smooth boundaries. These conditions can probably be relaxed, but the proofs would become rather involved. The necessary estimates of the Green function are proved in an Appendix.

\section{$\S 2$. Construction of the Functional Measures}

Let us consider a bounded domain $\Lambda \subset \mathbb{R}^{v}$, whose boundary $\partial \Lambda$ consists of a finite number of connected components which are $(v-1)$ dimensional surfaces of class $\boldsymbol{C}^{3}$. We are concerned with the Green function of the heat equation

$$
\frac{\partial u}{\partial t}(t, x)=\frac{1}{2} \Delta_{x} u(t, x) ; \quad t>0, \quad x \in \Lambda,
$$

with boundary condition:

or:

$$
u(t, x)=0 ; \quad t>0, \quad x \in \partial \Lambda
$$

$$
\frac{\partial u}{\partial n_{x}}(t, x)=\sigma(x) u(t, x) ; \quad t>0, \quad x \in \partial \Lambda,
$$

where $\partial / \partial n_{x}$ denotes the derivative along the inner normal, and $\sigma$ is a non-negative $C^{3}$-function on $\partial A$. Both boundary conditions will be considered together putting formally $\sigma=\infty$ when (2.2a) is to be understood.

The following two theorems summarize the information needed about the Green function.

Theorem 1 [6]. (i) There is one and only one function, $G_{\sigma}\left(t, x ; t^{\prime}, x^{\prime}\right)$, (named the Green function of (2.1), (2.2)), defined for $x, x^{\prime} \in \bar{\Lambda}$ and $t>t^{\prime}$ (depending in fact only on $t-t^{\prime}$ ), continuous on $\bar{\Lambda} \times \bar{\Lambda}$ for every fixed $t>t^{\prime}$ and having the following property:

For every continuous $f: \bar{\Lambda} \rightarrow \mathbb{R}$,

$$
u(t, x)=\int_{\Lambda} G_{\sigma}\left(t, x ; t^{\prime}, x^{\prime}\right) f\left(x^{\prime}\right) d x^{\prime}
$$

is the unique solution of Eqs. (2.1), (2.2) satisfying the initial condition:

$$
\lim _{t \searrow t^{\prime}} u(t, x)=f(x) \quad \text { uniformly in } \bar{\Lambda} \text {. }
$$

(ii) $G_{\sigma}\left(t, x ; t^{\prime}, x^{\prime}\right) \geqq 0 ; \quad t>t^{\prime}, \quad x, x^{\prime} \in \bar{\Lambda}$,

(iii) $\int_{\Lambda} G_{\sigma}(t, x ; \tau, y) G_{\sigma}\left(\tau, y ; t^{\prime}, x^{\prime}\right) d y=G_{\sigma}\left(t, x ; t^{\prime}, x^{\prime}\right)$;

$$
t>\tau>t^{\prime}, \quad x, x^{\prime} \in \bar{\Lambda},
$$

(iv) $\int_{\Lambda} G_{\sigma}\left(t, x ; t^{\prime}, x^{\prime}\right) d x \leqq 1 ; \quad t>t^{\prime}, \quad x^{\prime} \in \bar{\Lambda}$.

$\sigma(x) \equiv 0$ implies equality. 
Let us denote

$$
G^{0}\left(t, x ; t^{\prime}, x^{\prime}\right)=\left[2 \pi\left(t-t^{\prime}\right)\right]^{-v / 2} \exp \left[-\frac{\left|x-x^{\prime}\right|^{2}}{2\left(t-t^{\prime}\right)}\right]
$$

the Green function for the whole space, and define the compensating Green function for (2.1), (2.2), $Z_{\sigma}$, by:

$$
G_{\sigma}\left(t, x ; t^{\prime}, x^{\prime}\right)=G^{0}\left(t, x ; t^{\prime}, x^{\prime}\right)-Z_{\sigma}\left(t, x ; t^{\prime}, x^{\prime}\right) .
$$

Theorem 2 [7]. For every $T>0$, there are constants $C, c^{\prime}, c^{\prime \prime}>0$, such that, for all $0<t-t^{\prime} \leqq T$ and $x, x^{\prime} \in \Lambda$

$$
\left|Z_{\sigma}\left(t, x ; t^{\prime}, x^{\prime}\right)\right| \leqq C G^{0}\left(\frac{t}{c^{\prime}}, x ; \frac{t^{\prime}}{c^{\prime}}, x\right) \exp \left[-c^{\prime \prime} \frac{l_{x^{\prime}}^{2}}{2\left(t-t^{\prime}\right)}\right]
$$

where $l_{x^{\prime}}$ is the distance from $x^{\prime}$ to $\partial \Lambda$.

The estimate (2.5) is sufficient for the purpose of this section. It will become however important later to know that constants $C, c^{\prime}$ and $c^{\prime \prime}$ can be chosen independent of the domain $\Lambda$ and to give some more information on the behaviour of $C$ for $T \rightarrow \infty$. A result of this kind will be proved in the Appendix.

We shall now outline the construction of the functional measures related to the Green functions for $\Lambda$, using the (by now) classical approach presented in the Appendix of [5].

The trajectory space will be:

$$
\Omega_{\Lambda, \beta}=\prod_{0 \leqq t \leqq \beta} \bar{\Lambda}_{t} \text { where } \bar{\Lambda}_{t}=\bar{\Lambda} \text { for all } t .
$$

$\Omega_{\Lambda, \beta}$ is a compact space in the Tychonoff topology. The Banach algebra of all continuous real functions on $\Omega_{\Lambda, \beta}$ with the uniform norm will be denoted $\mathscr{C}\left(\Omega_{\Lambda, \beta}\right)$. The set of all the functions $\varphi: \Omega_{\Lambda, \beta} \rightarrow \mathbb{R}$, such that there exist $0<t_{1}<\cdots<t_{n}<\beta$ and a continuous function $F: \overline{\Lambda^{n}} \rightarrow \mathbb{R}$ for which:

$$
\varphi(\omega)=F\left(\omega\left(t_{1}\right), \ldots, \omega\left(t_{n}\right)\right), \quad \omega \in \Omega_{\Lambda, \beta}
$$

will be denoted $\mathscr{C}_{\text {fin }}\left(\Omega_{\Lambda, \beta}\right)$, and is a dense subalgebra of $\mathscr{C}\left(\Omega_{\Lambda, \beta}\right)$. For every $x, y \in \bar{\Lambda}$, a linear functional on $\mathscr{C}_{f \text { in }}\left(\Omega_{\Lambda, \beta}\right)$ will be defined through:

$$
\begin{aligned}
P_{x y}^{\sigma, \beta}(\varphi)= & \int_{\Lambda} d x_{1} G_{\sigma}\left(t_{1}, x_{1} ; 0, x\right) \int_{\Lambda} d x_{2} G_{\sigma}\left(t_{2}, x_{2} ; t_{1}, x_{1}\right) \cdots \times \\
& \times \int_{\Lambda} d x_{n} G_{\sigma}\left(t_{n}, x_{n} ; t_{n-1}, x_{n-1}\right) G_{\sigma}\left(\beta, y ; t_{n}, x_{n}\right) F\left(x_{1}, \ldots, x_{n}\right)
\end{aligned}
$$

where $\varphi$ and $F$ are related by (2.6).

The consistency of this definition follows from the semigroup property of $G_{\sigma}$ (Theorem 1, (iii)). Clearly, $\varphi \geqq 0$ implies $P_{x y}^{\sigma, \beta}(\varphi) \geqq 0$, because of the non-negativity of $G_{\sigma}$ (Theorem 1, (ii)). By a standard argument, 
this implies the boundedness of $P_{x y}^{\sigma, \beta}$, which can thus be extended by continuity to $\mathscr{C}\left(\Omega_{\Lambda, \beta}\right)$. The Riesz theorem [8] asserts the existence of a unique regular positive measure, $P_{x y}^{\sigma, \beta}(\cdot)$, defined on all Borel subsets of $\Omega_{\Lambda, \beta}$, such that:

$$
P_{x y}^{\sigma, \beta}(\varphi)=\int_{\Omega_{\Lambda, \beta}} P_{x y}^{\sigma, \beta}(d \omega) \varphi(\omega), \quad \varphi \in \mathscr{C}\left(\Omega_{\Lambda, \beta}\right) .
$$

\section{Remarks}

$1^{\circ}$. We shall denote $G_{N}\left(t, x ; t^{\prime}, x^{\prime}\right)$ the Green function for Neumann boundary conditions $(\sigma(x) \equiv 0$ in $(2.2 \mathrm{~b}))$. In view of Theorem 1 , (iv), $G_{N}\left(t, x ; t^{\prime}, x^{\prime}\right) d x$ are probability measures on $\bar{\Lambda}$ for all $t>t^{\prime}, x^{\prime} \in \bar{\Lambda}$, and can be interpreted as the transition probabilities of a stochastic process. Then, the unconditional measure $P_{x}^{N}$ can be defined as well.

$2^{\circ}$. The standard way of writing the solution of the heat equation with non-homogeneous boundary conditions in terms of the Green function [6] allows a proof of the following ordering of the Green functions:

$$
\begin{aligned}
& \sigma_{1}(x) \leqq \sigma_{2}(x) \text { for all } x \in \partial \Lambda \text { implies, } \\
& G_{\sigma_{1}}\left(t, x ; t^{\prime}, x^{\prime}\right) \geqq G_{\sigma_{2}}\left(t, x ; t^{\prime}, x^{\prime}\right) \text { for all } x, x^{\prime} \in \bar{\Lambda} .
\end{aligned}
$$

In particular, $G_{\infty}$ is dominated by and $G_{N}$ dominates all $G_{\sigma}$ in the considered class. This implies that the same ordering remains true for the functional measures, in particular:

$$
P_{x y}^{\infty, \beta}(A) \leqq P_{x y}^{\sigma, \beta}(A) \leqq P_{x y}^{N, \beta}(A) ; \quad \boldsymbol{A} \text { a Borel set in } \Omega_{\Lambda, \beta}
$$

$3^{\circ}$. All measures $P_{x y}^{\sigma, \beta}$ are concentrated on the set of $\alpha$-Hölder continuous trajectories for every $0<\alpha<1 / 2$, which start from $x$ at $t=0$ and finish in $y$ at $t=\beta$. In view of Remark $2^{\circ}$ above, it is sufficient to prove that the complementary set is $P_{x y}^{N, \beta}$-negligible. Because of Remark $1^{\circ}$ and Theorem 2, this proof can be taken over without essential modifications from $[5,9]$.

Let $P_{x y}^{W, \beta}$ be the conditional Wiener measure and

$$
\alpha_{\Lambda}(\omega)= \begin{cases}1 & \text { if } \omega(t) \in \Lambda \text { for all } t \in[0, \beta] \\ 0 & \text { otherwise }\end{cases}
$$

As $\alpha_{A}$ is a $P_{x y}^{W, \beta}$-measurable function, $\alpha_{A}(\omega) P_{x y}^{W, \beta}(d \omega)$ is a measure on $\Omega_{\Lambda, \beta}$. By the same argument, $\alpha_{\Lambda}(\omega) P_{x y}^{\sigma, \beta}(d \omega)$ are measures on $\Omega_{\Lambda, \beta}$.

Our main result concerning the family of measures constructed above is the following: 
Proposition 1. For every Borel subset $A \subset \Omega_{\Lambda, \beta}$ :

$$
\int_{A} P_{x y}^{\sigma, \beta}(d \omega) \alpha_{A}(\omega)=\int_{A} P_{x y}^{W, \beta}(d \omega) \alpha_{A}(\omega) .
$$

Proof. We shall first consider the case $A=\Omega_{\Lambda, \beta}$. Let $K_{n} \subset \Lambda$ be an increasing sequence of compact sets whose union is $\Lambda$. Let:

$$
\alpha_{K n}(\omega)= \begin{cases}1 & \text { if } \omega(t) \in K_{n} \text { for all } t \in[0, \beta] \\ 0 & \text { otherwise }\end{cases}
$$

Because every continuous trajectory which does not touch $\partial \Lambda$ has compact image in $\Lambda$, we shall have:

$$
\lim _{n \rightarrow \infty} \alpha_{K_{n}}(\omega)=\alpha_{A}(\omega), \quad P_{x y}^{\sigma, \beta}-\text { and } P_{x y}^{W, \beta}-\text { a.e. }
$$

In view of the Lebesgue dominated convergence theorem [8], it will be sufficient to show that:

$$
\int_{\Omega_{\Lambda, \beta}} P_{x y}^{\sigma, \beta}(d \omega) \alpha_{K}(\omega)=\int_{\Omega_{\mathbb{R}^{v}, \beta}} P_{x y}^{W, \beta}(d \omega) \alpha_{K}(\omega)
$$

with $K \subset \Lambda$ an arbitrary compact. Let $d=\operatorname{dist}(K, \partial \Lambda)$. Let $n \geqq 1$, and $t_{k}=\frac{k \beta}{n+1}, k=0,1, \ldots, n+1$. Let

$$
\alpha_{n}(\omega)= \begin{cases}1 & \text { if } \omega\left(t_{k}\right) \in K \text { for all } k=0,1, \ldots, n+1 \\ 0 & \text { otherwise }\end{cases}
$$

We have $\lim _{n} \alpha_{n}(\omega)=\alpha_{K}(\omega)$. Now:

$$
\begin{aligned}
\int_{\Omega_{\Lambda, \beta}} P_{x y}^{\sigma, \beta}(d \omega) \alpha_{n}(\omega)= & \int_{K^{n}} d x_{1} \ldots d x_{n} \prod_{k=0}^{n}\left[G^{0}\left(t_{k+1}, x_{k+1} ; t_{k}, x_{k}\right)\right. \\
& \left.-Z_{\sigma}\left(t_{k+1}, x_{k+1} ; t_{k}, x_{k}\right)\right] \\
= & \int_{K^{n}} d x_{1} \ldots d x_{n} \prod_{k=0}^{n} G^{0}\left(t_{k+1}, x_{k+1} ; t_{k}, x_{k}\right)+R_{n} \\
= & \int_{\Omega_{\mathbb{R}^{\prime}, \beta}} P_{x y}^{W, \beta}(d \omega) \alpha_{n}(\omega)+R_{n}
\end{aligned}
$$

where $x_{0}=x, x_{n+1}=y$, and we shall show that $\lim _{n} R_{n}=0$.

The general term in $R_{n}$ has the form:

$$
\pm \int_{K^{n}} d x_{1} \ldots d x_{n}\left[\ldots G^{0} G^{0} \ldots G^{0} Z_{\sigma} G^{0} \ldots G^{0} Z_{\sigma} Z_{\sigma} G^{0} \ldots\right]
$$


with $k+1$ factors $Z$ and $n-k$ factors $G^{0}$. Taking moduli, extending to $\mathbb{R}^{v}$ all integrals which do not involve $Z$-factors and using the semigroup property of $G^{0}$, this will be majorized by an integral containing $k+1$ factors $|Z|$ and at most $k+2$ factors $G^{0}$. With the inequality (2.5), $l_{x_{k}} \geqq d$ and

$$
G^{0}\left(t, x ; t^{\prime}, x^{\prime}\right) \leqq c^{\prime-v / 2} G^{0}\left(\frac{t}{c^{\prime}}, x ; \frac{t^{\prime}}{c^{\prime}}, x^{\prime}\right) \quad\left(0<c^{\prime} \leqq 1\right)
$$

one obtains, after extending again the integrals to $\mathbb{R}^{v}$ :

$$
\begin{aligned}
&\left|R_{n}\right| \leqq c^{\prime-v / 2} G^{0}\left(\frac{\beta}{c^{\prime}}, y ; 0, x\right) \sum_{k=0}^{n} \frac{(n+1) !}{(k+1) !(n-k) !} \\
& \\
& \cdot\left[C c^{\prime-v / 2} \exp \left(-c^{\prime \prime} \frac{d^{2}(n+1)}{2 \beta}\right)\right]^{k+1} \\
&=c^{\prime-v / 2} G^{0}\left(\frac{\beta}{c^{\prime}}, y ; 0, x\right) \\
& \cdot\left\{\left[1+C c^{\prime-v / 2} \exp \left(-c^{\prime \prime} \frac{d^{2}(n+1)}{2 \beta}\right)\right]^{n+1}-1\right\} \underset{\substack{\rightarrow \rightarrow \infty \\
n \rightarrow \infty}}{ }
\end{aligned}
$$

If $\boldsymbol{A} \subset \Omega_{\Lambda, \beta}$ is an arbitrary Borel set, there is a sequence of functions in $\mathscr{C}_{\text {fin }}\left(\Omega_{A, \beta}\right)$ converging to its characteristic function, and it will be sufficient to prove:

$$
\int_{\Omega_{\Lambda, \beta}} P_{x y}^{\sigma, \beta}(d \omega) \alpha_{\Lambda}(\omega) f(\omega)=\int_{\Omega_{\mathbb{R}^{v}, \beta}} P_{x y}^{W, \beta}(d \omega) \alpha_{\Lambda}(\omega) f(\omega), \quad f \in \mathscr{C}_{\mathrm{fin}}\left(\Omega_{\Lambda, \beta}\right),
$$

But this follows at once from the first part of the proof and from:

$$
\begin{aligned}
& \int_{\Omega_{\Lambda, \beta}} P_{x y}^{\sigma, \beta}(d \omega) \alpha_{\Lambda}(\omega) f(\omega)=\int_{\Lambda^{n}} d x_{1} \ldots d x_{n} F\left(x_{1}, \ldots, x_{n}\right) \\
& \quad \int_{\Omega_{\Lambda, t_{1}}} P_{x x_{1}}^{\sigma, t_{1}}\left(d \omega_{1}\right) \alpha_{\Lambda}\left(\omega_{1}\right) \ldots \int_{\Omega_{\Lambda, \beta-t_{n}}} P_{x_{n} y}^{\sigma, \beta-t_{n}}\left(d \omega_{n+1}\right) \alpha_{\Lambda}\left(\omega_{n+1}\right)
\end{aligned}
$$

where $f$ and $F$ are related by (2.6), and the similar equality for the Wiener measure. This finishes the proof.

As expected, for the appropriate particular cases we reobtain the measures considered by Ginibre [3] and Novikov [4].

Corollary. For every Borel subset $\boldsymbol{A} \subset \Omega_{\Lambda, \beta}$ and $x, y \in \bar{\Lambda}$

$$
P_{x y}^{\infty, \beta}(\boldsymbol{A})=\int_{A} P_{x y}^{W, \beta}(d \omega) \alpha_{\Lambda}(\omega)
$$


Proof. In view of Proposition 1, one has only to show that the $P_{x y}^{\infty, \beta_{-}}$ measure of the set $B$ of trajectories inside $\bar{A}$ which do touch $\partial \Lambda$ for at least one $t \in[0, \beta]$ is zero. But this is a consequence of:

$$
P_{x y}^{\infty, \beta}(A) \leqq A_{x y}^{W, \beta}(A) \quad \text { for every measurable } A \subset \Omega_{\Lambda, \beta}
$$

which follows from a corresponding inequality for Green functions, and of:

$$
P_{x y}^{W, \beta}(\boldsymbol{B})=0
$$

which is a well known result on conditional Wiener measure [9].

\section{Remark}

$4^{\circ}$. This latter property of the Wiener measure is shared also by all the measures considered here, provided the smooth surface to be touched but not crossed is required to be a compact subset of $\Lambda$. It is sufficient to consider $P_{x y}^{N, \beta}$, where, using Remark $1^{0}$, essentially the same proof as for conditional Wiener measure applies.

Let $L>0$, and $\varepsilon: \mathbb{R} \rightarrow\left[-\frac{L}{2}, \frac{L}{2}\right]$ defined by:

$\varepsilon(x)=\left\{\begin{array}{ll}x-2 n L & \text { if }\left(2 n-\frac{1}{2}\right) L \leqq x \leqq\left(2 n+\frac{1}{2}\right) L, n=0, \pm 1, \ldots \\ (2 n+1) L-x & \text { if }\left(2 n+\frac{1}{2}\right) L \leqq x \leqq\left(2 n+\frac{3}{2}\right) L, n=0, \pm 1, \ldots\end{array}\right.$.

The Green function for Neumann boundary conditions on $\left[-\frac{L}{2}, \frac{L}{2}\right]$ is:

$$
G_{N}\left(t, x ; t^{\prime}, x^{\prime}\right)=\sum_{u \in \varepsilon^{-1}\left(x^{\prime}\right)} G^{0}\left(t, x ; t^{\prime}, u\right) .
$$

If $\varepsilon^{\prime}: \Omega_{\mathbb{R}, \beta} \rightarrow \Omega\left[-\frac{L}{2}, \frac{L}{2}\right], \beta$ is defined by $\left[\varepsilon^{\prime}(\omega)\right](t)=\varepsilon(\omega(t))$, then:

$$
\tilde{P}_{x y}^{\beta}(A) \equiv \sum_{u \in \varepsilon^{-1}(y)} P_{x u}^{W, \beta}\left(\varepsilon^{\prime-1}(A)\right), \quad A \text { measurable }
$$

is the measure employed by Novikov [4].

Proposition 2. For every Borel subset $A \subset \Omega\left[-\frac{L}{2}, \frac{L}{2}\right], \beta$ and $x, y \in \bar{\Lambda}$, $P_{x y}^{N, \beta}(A)=\tilde{P}_{x y}^{\beta}(A)$.

Proof. If $\boldsymbol{A}=\Omega\left[-\frac{L}{2}, \frac{L}{2}\right], \beta$, this is just (2.15). We shall now prove:

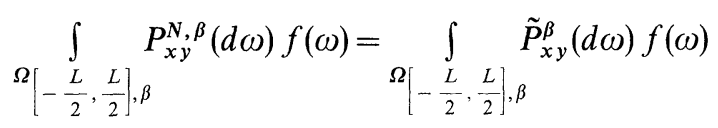

for all $f(\omega)=F\left(\omega\left(t_{1}\right), \ldots, \omega\left(t_{n}\right)\right) \in \mathscr{C}_{\text {fin }}\left(\Omega\left[-\frac{L}{2}, \frac{L}{2}\right], \beta\right)$. 
For every continuous function $h:\left[-\frac{L}{2}, \frac{L}{2}\right] \rightarrow \mathbb{R}$ and $z \in \mathbb{R}$

$$
\int_{\mathbb{R}} d v G^{0}\left(t, v ; t^{\prime}, z\right) h(\varepsilon(v))=\int_{-\frac{L}{2}}^{\frac{L}{2}} d w G_{N}\left(t, w ; t^{\prime}, \varepsilon(z)\right) h(w) .
$$

Now, using (2.16), (2.15), and (2.18), one obtains

$$
\begin{aligned}
\tilde{P}_{x y}^{\beta}(f)= & \sum_{\left.u \in \varepsilon^{-1}(y)\right)} \int_{\mathbb{R}^{n}} d v_{1} \ldots d v_{n} G^{0}\left(t_{1}, v_{1} ; 0, x\right) G^{0}\left(t_{2}, v_{2} ; t_{1}, v_{1}\right) \ldots \\
& \times G^{\circ}\left(\beta, u ; t_{n}, v_{n}\right) F\left(\varepsilon\left(v_{1}\right), \ldots, \varepsilon\left(v_{n}\right)\right) \\
= & \int_{\mathbb{R}^{n}} d v_{1} \ldots d v_{n} G^{0}\left(t_{1}, v_{1} ; 0, x\right) G^{0}\left(t_{2}, v_{2}, t_{1}, v_{1}\right) \ldots \\
& \times G_{N}\left(\beta, y ; t_{n}, \varepsilon\left(v_{n}\right)\right) F\left(\varepsilon\left(v_{1}\right), \ldots, \varepsilon\left(v_{n}\right)\right) \\
= & \int\left[-\frac{L}{2}, \frac{L}{2}\right]^{n} d w_{1} \ldots d w_{n} G_{N}\left(t_{1}, w_{1} ; 0, x\right) G_{N}\left(t_{2}, w_{2} ; t_{1}, w_{1}\right) \ldots \\
& \times G_{N}\left(\beta, y ; t_{n}, w_{n}\right) F\left(w_{1}, \ldots, w_{n}\right) \\
= & P_{x y}^{N, \beta}(f),
\end{aligned}
$$

which finishes the proof.

\section{Remark}

$5^{\circ}$. If $\Lambda$ is a parallelepiped, periodic boundary conditions can be imposed for Eq. (2.1), as well. The Green function can be written in terms of $G^{0}$ as follows:

$$
G_{\mathrm{per}}\left(t, x ; t^{\prime}, x^{\prime}\right)=\sum_{u \in \eta^{-1}\left(x^{\prime}\right)} G^{\circ}\left(t, x ; t^{\prime}, u\right),
$$

where $\eta: \mathbb{R}^{v} \rightarrow \Lambda$ is defined in the following way: Divide the whole $\mathbb{R}^{v}$ into translates of $\Lambda ; \eta$ restricted to one such translate means the inverse translation. Theorem 1 is still true in this case and also the estimate in Theorem 2, if $\left|x-x^{\prime}\right|$ is replaced by the distance between $x$ and $x^{\prime}$ in $\bar{\Lambda}$ considered as a torus. Therefore, $G_{\mathrm{per}}$ defines a measure $P_{x y}^{\mathrm{per}, \beta}$ on $\Omega_{\Lambda, \beta}$, which now will be concentrated on the set of continuous trajectories in $\bar{\Lambda}$ with the torus topology. In the same way as in Proposition 2, one can see that this measure is given by:

$$
P_{x y}^{\text {per, }, \beta}(A)=\sum_{u \in \eta^{-1}(y)} P_{x u}^{W, \beta}\left(\eta^{\prime-1}(A)\right) \quad \text { for } \quad A \subset \Omega_{\Lambda, \beta} .
$$




\section{§ 3. The Thermodynamic Pressure}

Suppose that the system consists of $n$ identical particles, obeying Boltzmann or Bose statistics ${ }^{1}$, enclosed in the bounded domain $\Lambda$ and interacting via a two-body potential $\Phi: \mathbb{R}^{v} \rightarrow \mathbb{R}$ satisfying the following conditions ${ }^{2}$.

(A) $\Phi(x)$ is an even function of $x$, bounded from below: $\Phi(x) \geqq-b$, and continuous outside a closed set of Newtonian capacity zero. If the potential has a hard-core: $\Phi(x)=+\infty$ for $|x| \leqq a, a>0$, continuity outside the core will be required. Below, both cases will be treated together, taking $a=0$ for potentials without hard-core.

(B) The interaction is stable, i.e. there exists $B \geqq 0$ such that for any finite family of points $x_{1}, \ldots, x_{m} \in \mathbb{R}^{v}$

$$
\sum_{i<j} \Phi\left(x_{i}-x_{j}\right) \geqq-m B \text {. }
$$

We shall in fact need the following much stronger condition:

$\left(\mathrm{B}_{1}\right)$ There exists a $B \geqq 0$, such that, for any finite family of points $x_{0}, x_{1}, \ldots, x_{m} \in \mathbb{R}^{v}$ with $\left|x_{i}-x_{j}\right| \geqq a, i \neq j$

$$
\sum_{i=1}^{m} \Phi\left(x_{0}-x_{i}\right) \geqq-2 B
$$

Note that if $a=0$, then $\left(\mathrm{B}_{1}\right)$ implies the positivity of $\Phi$.

(C) There exists $R>0$, such that $\Phi(x) \leqq 0$ for $|x| \geqq R$.

Let $\Omega_{\Lambda^{n}, \beta}$ be defined as in Section 2, and:

$$
\alpha(\omega)=\alpha\left(\omega^{1}, \ldots, \omega^{n}\right)=\left\{\begin{array}{rr}
1 & \text { if } \quad\left|\omega^{i}(t)-\omega^{j}(t)\right|>a \\
& \text { for all } t \in[0, \beta], \quad i \neq j=1, \ldots, n \\
0 & \text { otherwise }
\end{array}\right.
$$

As usually, we consider the following integral:

$$
W^{\beta}(x, y)=\int_{\Omega_{\Lambda^{n}, \beta}} P_{x y}^{\sigma, \beta}(d \omega) \alpha(\omega) \exp \left[-\int_{0}^{\beta} d t U(\omega(t))\right],
$$

where $x=\left(x^{1} \ldots, x^{n}\right), y=\left(y^{1}, \ldots, y^{n}\right) \in \bar{\Lambda}^{n} ; U(\omega(t))=\sum_{i<j} \Phi\left(\omega^{i}(t)-\omega^{j}(t)\right)$; and $P_{x y}^{\sigma, \beta}(d \omega)=\prod_{i=1}^{n} P_{x^{i} y^{i}}^{\sigma, \beta}\left(d \omega^{i}\right)$ with $P_{x^{i} y^{i}}^{\sigma, \beta}$ defined in Section 2 .

We shall denote:

$$
S_{a}=\left\{x \in \overline{\Lambda^{n}}|| x^{i}-x^{j} \mid>a, \forall i \neq j=1,2, \ldots, n\right\}
$$

and $\mathfrak{S}=L^{2}\left(\overline{\Lambda^{n}} \cap S_{a}\right)$ the Hilbert space of the system.

\footnotetext{
${ }^{1}$ It seems that the functional integral approach is not suited to handle easily Fermi statistics [9].

${ }^{2}$ We list here all conditions to be used below. It should be remarked however that only condition (A) is used for writing the Feynman-Kac formula. Besides, condition (C) can be relaxed for the purposes of this paper.
} 
In view of (A) and the definition (3.1), the integrand in (3.2) is defined a.e. on $S_{a} \times S_{a}$ with respect to $P_{x y}^{\sigma, \beta}$ and the integral converges.

The following facts can be proved in the same way as in [3]:

$W^{\beta}(x, y)$ is a continuous function of $(x, y) \in S_{a} \times S_{a}$. The kernel $W^{\beta}(x, y)$ defines a bounded selfadjoint positive trace-class operator of $\mathfrak{H}$. $W^{\beta}(x, y)$ has the semigroup property:

and

$$
\int_{S_{a}} d z W^{\beta}(x, z) W^{\beta^{\prime}}(z, y)=W^{\beta+\beta^{\prime}}(x, y), \quad x, y \in S_{a}
$$

$$
\|\| W^{\beta} \| \leqq \exp [-\beta \inf U(x)] \text {. }
$$

Moreover, $W^{\beta}, \beta \geqq 0$ is a strongly continuous semigroup and its infinitesimal generator $H$ restricted to functions of $C^{2}$ class of compact support in $\Lambda^{n} \cap S_{a}$ is given by $-\frac{1}{2} \Delta+U$.

Using (3.2), integral representations of the grandcanonical partition function can be written down $[3,9]$. We shall consider only the case of Bose statistics, because the case of Maxwell-Boltzmann statistics is simpler. Then:

$$
\begin{aligned}
\Xi_{\Lambda, \sigma}(z, \beta)= & \sum_{n=0}^{\infty} \frac{1}{n !} \sum_{j_{1}=1}^{\infty} \cdots \sum_{j_{n}=1}^{\infty} \frac{z^{j_{1}+\cdots+j_{n}}}{j_{1} \ldots j_{n}} \int_{A} d u_{1} \int P_{u_{1}, u_{1}}^{\sigma, j_{1} \beta}\left(d \tilde{\omega}_{1}\right) \ldots \\
& \times \int_{\Lambda} d u_{n} \int P_{u_{n} u_{n}}^{\sigma, j_{n} \beta}\left(d \tilde{\omega}_{n}\right) \exp \left[-\tilde{u}\left(\tilde{\omega}_{1}, \ldots, \tilde{\omega}_{n}\right)\right],
\end{aligned}
$$

where $\tilde{\omega}_{i}$ are closed loops of time interval $j_{i} \beta$, obtained by putting together the elementary trajectories $\omega_{i k}:[0, \beta] \rightarrow \bar{\Lambda}, k=1, \ldots, j_{i}, i=1, \ldots, n$ and:

$$
\tilde{u}\left(\tilde{\omega}_{1}, \ldots, \tilde{\omega}_{n}\right)=\int_{0}^{\beta} d t U\left(\omega_{11}(t), \ldots, \omega_{1 j_{1}}(t), \ldots, \omega_{n 1}(t), \ldots, \omega_{n j_{n}}(t)\right) .
$$

The finite-volume pressure is:

$$
p_{\Lambda, \sigma}(z, \beta)=\frac{1}{\beta V(\Lambda)} \log \Xi_{\Lambda, \sigma}(z, \beta) .
$$

For Dirichlet boundary conditions, the thermodynamic limit is known to exist under conditions (A), (B), (C) on $\Phi$ [9]. The existence of the thermodynamical limit for other boundary conditions and its equality to the limit for Dirichlet conditions results from the following:

Proposition 3. Let $\Lambda_{n}$ be a sequence of convex bounded domains in $\mathbb{R}^{v}, \Lambda_{n} \rightarrow \infty$ (Fisher), whose boundaries $\partial \Lambda_{n}$ are $\boldsymbol{C}^{3}$-surfaces with uniformly bounded mean curvature. Let $\sigma_{n}: \partial \Lambda_{n} \rightarrow \mathbb{R}_{+}$, a sequence of functions of class $C^{3}$. Then, for interactions satisfying $(\mathrm{A}),\left(\mathrm{B}_{1}\right),(\mathrm{C})$, and for $0<z<e^{-2 B \beta}$.

$$
\lim _{n}\left[p_{\Lambda_{n}, \sigma_{n}}(z, \beta)-p_{\Lambda_{n}, \infty}(z, \beta)\right]=0 .
$$

Proof. The ordering (2.9) implies, for $z>0$,

$$
p_{A_{n}, \infty}(z, p) \leqq p_{A_{n}, \sigma_{n}}(z, \beta) \leqq p_{\Lambda_{n}, N}(z, \beta) \text {. }
$$


Then, splitting the functional integrals in (3.6) into the sum of the integrals over the trajectories which do and which do not touch $\partial \Lambda_{n}$, using Proposition 1, condition $\left(\mathrm{B}_{1}\right)$ and rearranging the terms, one obtains:

$$
\begin{aligned}
& p_{\Lambda_{n}, N}(z, \beta)-p_{\Lambda_{n}, \infty}(z, \beta) \\
& \quad \leqq \frac{1}{\beta V\left(\Lambda_{n}\right)} \sum_{j=1}^{\infty} \frac{\left(z e^{2 B \beta}\right)^{j}}{j} \int_{\Lambda_{n}} d u \int_{\Omega_{\Lambda_{n}, j \beta}} P_{u u}^{N, j \beta}(d \tilde{\omega})\left[1-\alpha_{\Lambda_{n}}(\tilde{\omega})\right] .
\end{aligned}
$$

Now

therefore

$$
\begin{aligned}
\int_{\Omega \Lambda_{n, j \beta}} P_{u u}^{N, j \beta}(d \tilde{\omega})\left[1-\alpha_{\Lambda_{n}}(\tilde{\omega})\right] & =Z_{\infty}(j \beta, u ; 0, u)-Z_{N}(j \beta, u ; 0, u) \\
& =Z_{\infty}(j \beta, u ; 0, u)+\mid Z_{N}(j \beta, u ; 0, u)
\end{aligned}
$$

$$
\begin{aligned}
p_{\Lambda_{n}, N}(z, \beta)-p_{\Lambda_{n}, \infty}(z, \beta) & \leqq \frac{1}{\beta V\left(\Lambda_{n}\right)} \sum_{j=1}^{\infty} \frac{\left(z e^{2 B \beta}\right)^{j}}{j} \int_{\Lambda_{n}} d u Z_{\infty}(j \beta, u ; o, u) \\
& +\frac{1}{\beta V\left(\Lambda_{n}\right)} \sum_{j=1}^{\infty} \frac{\left(z e^{2 B \beta}\right)^{j}}{j} \int_{\Lambda_{n}} d u\left|Z_{N}(j \beta, u ; 0, u)\right|
\end{aligned}
$$

Denoting $S_{n}(d)$ the area of the surface $\left\{x \in \Lambda_{n} \mid \operatorname{dist}\left(x, \partial \Lambda_{n}\right)=d\right\}$ and $S_{n}=\operatorname{area}\left(\partial \Lambda_{n}\right)$, the convexity of $\Lambda_{n}$ implies

$$
S_{n}(d) \leqq S_{n}
$$

Using Proposition A1 and (3.11), one obtains:

$$
\int_{A_{n}} d u Z_{\infty}(j \beta, u ; 0, u) \leqq e^{v / 2} \frac{1}{2(2 \pi j \beta)^{\frac{v-1}{2}}} S_{n}
$$

wherefrom the first series in (3.10) is majorized by:

$$
\frac{e^{v / 2}}{2(2 \pi \beta)^{\frac{v-1}{2}}} S_{n} \cdot g_{\frac{v-1}{2}}\left(z e^{2 B \beta}\right) .
$$

The second series in (3.10) can be majorized in the same way, using Proposition A. 2 but the resulting series converges only up to $e^{-(2 B+\lambda) \beta}$. This shows that, for $z<e^{-(2 B+\lambda) \beta}, p_{\Lambda_{n}, N}-p_{A_{n}, \infty}$ vanishes for $n \rightarrow \infty$ as $\frac{S_{n}}{V\left(\Lambda_{n}\right)}$.

The equality of thermodynamic pressures can also be proved for $e^{-(2 B+\lambda) \beta} \leqq z<e^{-2 B \beta}$, making use of Proposition A.3 as well. Namely, 
one truncates the second series in (3.10) at $j_{n}$, and uses (A.4) for $j \leqq j_{n}$ and (A.13) for $j>j_{n}$, to obtain:

$$
\begin{aligned}
& \frac{1}{\beta V\left(\Lambda_{n}\right)} \sum_{j=1}^{\infty} \frac{\left(z e^{2 B \beta}\right)^{j}}{j} \int_{\Lambda_{n}} d u\left|Z_{N}(j \beta, u ; 0, u)\right| \\
& \leqq \frac{S_{n}}{\beta V\left(\Lambda_{n}\right)} 2 C \beta^{-\frac{v-1}{2}} \sqrt{\frac{2 \pi}{c}} j_{n}\left[z e^{(2 B+\lambda) \beta}\right]^{j_{n}}+\frac{M(\beta)}{\beta} \cdot \frac{\left[z e^{2 B \beta}\right]^{j_{n}+1}}{1-z e^{2 B \beta}}
\end{aligned}
$$

which tends to zero for $n \rightarrow \infty$, if $j_{n} \rightarrow \infty$ in such a way that:

$$
\frac{S_{n}}{V\left(\Lambda_{n}\right)} j_{n}\left[z e^{(2 B+\lambda) \beta}\right]^{j_{n}} \rightarrow 0 \text {. }
$$

\section{Appendix}

In this appendix we shall give a proof of the estimates of the Green function needed in Section 3.

We consider first the case of the Dirichlet boundary condition, which is a simple application of the maximum principle.

Proposition A1.

$$
0 \leqq Z_{\infty}\left(t, x ; 0, x^{\prime}\right) \leqq e^{v / 2}(2 \pi t)^{-v / 2} e^{-\frac{l_{x^{\prime}}}{2 t}}
$$

Proof. $Z_{\infty}\left(t, x ; 0, x^{\prime}\right)$ is the solution of the heat Eq. (2.1), with vanishing initial condition and with boundary condition:

$$
Z_{\infty}\left(t, x ; 0, x^{\prime}\right)=G^{0}\left(t, x ; 0, x^{\prime}\right) ; \quad x \in \partial \Lambda, \quad t>0, \quad x^{\prime} \in \bar{\Lambda} .
$$

If $l_{x^{\prime}}^{2} \geqq t v$, we shall use for estimation the maximum principle:

$$
Z_{\infty}\left(t, x ; 0, x^{\prime}\right) \leqq \sup _{\substack{0<\tau \leqq t \\ y \in \partial A}} G^{0}\left(\tau, y ; 0, x^{\prime}\right) \leqq \sup _{0<\tau \leqq t}(2 \pi \tau)^{-v / 2} e^{-\frac{l_{x^{\prime}}}{2 \tau}} .
$$

This is an increasing function of $\tau$ for $l_{x^{\prime}}^{2} \geqq \tau v$, and then:

$$
Z_{\infty}\left(t, x ; 0, x^{\prime}\right) \leqq(2 \pi t)^{-v / 2} e^{-\frac{l l_{x^{\prime}}}{2 t}}
$$

If $l_{x^{\prime}}^{2}<t v$, the positivity of $G_{\infty}$ and $Z_{\infty}$ will be used, wherefrom:

$$
\begin{aligned}
0 \leqq Z_{\infty}\left(t, x ; 0, x^{\prime}\right) & \leqq G^{0}\left(t, x ; 0, x^{\prime}\right) \leqq(2 \pi t)^{-v / 2} \\
& \leqq e^{v / 2}(2 \pi t)^{-v / 2} e^{-\frac{l l^{\prime}}{2 t}} .
\end{aligned}
$$

Proposition A 2. Let $R>0$. Then, there are constants $\lambda \geqq 0, C>0$, $c>0$, such that, for every bounded convex domain $\Lambda \subset \mathbb{R}^{v}$, whose boundary 
$\partial \Lambda$ is a $C^{3}$ surface of mean curvature less than $1 / R$, the following inequality holds:

$$
Z_{N}\left(t, x ; 0, x^{\prime}\right) \leqq C e^{\lambda t} t^{-v / 2} \exp \left[-c \frac{l_{x}^{2}+l_{x,}^{2}}{2 t}\right] .
$$

Proof. For simplicity, we shall consider only the $v=3$ case.

From the integral equation for $Z_{N}$ [10], one obtains the following Neumann series solution:

$Z_{N}\left(t, x ; 0, x^{\prime}\right)=-2 \int_{0}^{t} d \sigma \int_{\partial \Lambda} d S_{\eta} G^{0}(t, x ; \sigma, \eta) \frac{\partial}{\partial n_{\eta}} G^{0}\left(\sigma, \eta ; 0, x^{\prime}\right)$
$-2 \sum_{k=1}^{\infty} \int_{0}^{t} d \sigma \int_{\partial \Lambda} d S_{\eta}\left\{\int_{\sigma}^{t} d \tau \int_{\partial \Lambda} d S_{\xi} \cdot G^{0}(t, x ; \tau, \xi) M_{k}(\tau, \xi ; \sigma, \eta)\right\} \frac{\partial}{\partial n_{\eta}} G^{0}\left(\sigma, \eta ; 0, x^{\prime}\right)$

where:

$$
\begin{gathered}
M_{1}(\tau, \xi ; \sigma, \eta)=-2 \frac{\partial}{\partial n_{\xi}} G^{0}(\tau, \xi ; \sigma, \eta) \\
M_{k+1}(\tau, \xi ; \sigma, \eta)=\int_{\sigma}^{\tau} d \theta \int_{\partial \Lambda} d S_{\zeta} M_{1}(\tau, \xi ; \theta, \zeta) M_{k}(\theta, \zeta ; \sigma, \eta) .
\end{gathered}
$$

Estimates for $Z_{N}$ will be obtained by term by term majorizations of the series (A.5) ${ }^{3}$. The proposition will follow from the inequality:

$$
\left|M_{k}(\tau, \xi ; \sigma, \eta)\right| \leqq \frac{C_{1}^{k}}{\Gamma\left(\frac{k}{2}\right)}(\tau-\sigma)^{\frac{k}{2}-2} \exp \left[-h \frac{|\xi-\eta|^{2}}{2(\tau-\sigma)}\right]
$$

which will be proved below. It should be emphasized that the constants $C_{1}, h$ do not depend on $A$.

First,

$$
\begin{aligned}
\left|M_{1}(\tau, \xi ; \sigma, \eta)\right| & =2 \frac{|\xi-\eta|}{\tau-\sigma} \cos \left(\vec{n}_{\xi}, \xi \vec{\eta}\right) G^{0}(\tau, \xi ; \sigma, \eta) \\
& \leqq \frac{|\xi-\eta|^{2}}{R(\tau-\sigma)} G^{0}(\tau, \xi ; \sigma, \eta),
\end{aligned}
$$

where the last inequality for the cosine of the angle between the inner normal to $\partial \Lambda, \vec{n}_{\xi}$, and the vector $\overrightarrow{\xi \eta}$ comes from the boundedness of the curvature, which implies the existence of a sphere of radius $R$, tangent to $\partial \Lambda$ in $\xi$ and contained in $\Lambda$. Using:

$$
\sup _{\substack{a>0 \\ t>0}} \frac{a^{\alpha}}{\mathrm{t}^{\alpha / 2}} e^{-\frac{a^{2}}{2 t}(1-h)}=\left[\frac{\alpha}{\mathrm{e}(1-\mathrm{h})}\right]^{\alpha / 2} ; \quad \alpha>0, \quad 0<h<1
$$

${ }^{3}$ As the series (A.5) is alternating, this majorization is very drastic, and is responsible alone for the factor $e^{\lambda t}$ appearing in (A.4). 
one can replace (A.8) by:

$$
\left|M_{1}(\tau, \xi ; \sigma, \eta)\right| \leqq \frac{C_{0}}{\Gamma\left(\frac{1}{2}\right)}(\tau-\sigma)^{-3 / 2} \exp \left[-h \frac{|\xi-\eta|^{2}}{2(\tau-\sigma)}\right]
$$

where $C_{0}$ depends only on $h$ and $R$.

In order to obtain the estimates (A.7) for the iterated kernels, we shall use an induction argument and, at every step, majorize the integral over $\partial \Lambda$ by an integral over $\mathbb{R}^{2}$, and make use of the following identity, which holds for every $\alpha, \beta \in(-\infty, 2)$ :

$$
\begin{gathered}
\int_{\sigma}^{\tau} d \theta \int_{\mathbb{R}^{2}} d^{2} \zeta(\tau-\theta)^{-\alpha}(\theta-\sigma)^{-\beta} \exp \left[-\frac{h|\xi-\zeta|^{2}}{2(\tau-\theta)}-\frac{h|\zeta-\eta|^{2}}{2(\theta-\sigma)}\right] \\
=\frac{2 \pi}{h} B(2-\alpha, 2-\beta)(\tau-\sigma)^{2-\alpha-\beta} \exp \left[-\frac{h|\xi-\eta|^{2}}{2(\tau-\sigma)}\right] .
\end{gathered}
$$

This will be done by applying parts of $\partial \Lambda$ on parts of planes containing the segment $\xi \eta$, in such a way that the surface element be not essentially diminished and $|\xi-\zeta|,|\zeta-\eta|$ become smaller for the image of $\zeta$. Let us describe this process in more detail:

a) One takes a coordinate system with the first axis along $\overrightarrow{\xi \eta}$ and consider:

$$
\begin{aligned}
& S_{1}=\left\{\zeta \in \partial \Lambda|| \alpha_{1}(\zeta) \mid \geqq \sqrt{\frac{5}{6}}\right\} \\
& S_{i}=\left\{\zeta \in \partial \Lambda|| \alpha_{i}(\zeta) \mid \geqq \frac{1}{2 \sqrt{3}}\right\}, \quad i=2,3,
\end{aligned}
$$

where $\alpha_{i}(\zeta)$ is the projection of $\vec{n}_{\zeta}$ on the $i$-th axis. Clearly, $S_{1} \cup S_{2} \cup S_{3}=\partial \Lambda$.

b) For $i=2,3$, both connected components of $S_{i}$ will be orthogonally projected on the coordinate plane perpendicular on the $i$-th axis. The surface element becomes at most $2 \sqrt{3}$ times smaller and distances decrease, therefore the integral over $S_{2} \cup S_{3}$ will be majorized by $8 \sqrt{3}$ times an integral over $\mathbb{R}^{2}$.

c) Let $S_{1}^{ \pm}$be the connected components of $S_{1}$ with $\pm \alpha_{1}(\zeta) \geqq \sqrt{\frac{5}{6}}$. We shall consider $S_{1}^{+} . S_{1}^{-}$can be treated in the same way. Because of the convexity of $\Lambda, S_{1}^{+}$lies entirely outside the circular cone with apex $\xi$ :

$$
\mathscr{C}=\left\{z \in \mathbb{R}^{3} \mid \nless(\overrightarrow{\xi z}, \overrightarrow{\xi \eta}) \leqq \arcsin \sqrt{\frac{5}{6}}<\frac{\pi}{2}\right\} .
$$

Let $\pi$ be a plane whose angle with $\overrightarrow{\xi \eta}$ is $\pi / 4 ; D_{1}, D_{2}$ the half-lines along which $\pi$ intersects $\partial \mathscr{C}$ and $\delta$ the intersection of $\pi$ with the 2,3-coordinate plane. Let $d_{1}, d_{2}$ be the orthogonal projections of $D_{1}, D_{2}$ on the 
2,3-coordinate plane and $\gamma=\Varangle\left(d_{1}, d_{2}\right)$. Let $S_{1}^{+}(\pi)$ be that piece of $S_{1}^{+}$ which is contained between the half-planes $\left(d_{1}, D_{1}\right)$ and $\left(d_{2}, D_{2}\right)$. A rigid rotation of $S_{1}^{+}(\pi)$ of angle $\pi / 2$ around $\delta$ will be performed, in which distances between $\zeta$ and $\xi$ are unaltered and distances between $\zeta$ and $\eta$ decrease. Then the rotated surface is orthogonally projected on the $(\delta, \overrightarrow{\xi \eta})$-plane, in which distances decrease and the surface element is diminished by at most a factor $\sqrt{\frac{5}{6}}$. The integral over $S_{1}^{+}(\pi)$ can then be replaced as above by $\sqrt{\frac{6}{5}}$ times an integral over $\mathbb{R}^{2}$. But $S_{1}^{+}$can be covered by at most $\left[\frac{2 \pi}{\gamma}\right]+1 S_{1}^{+}(\pi)$ 's. Therefore the integral over $S_{1}$ is majorized by $2 \sqrt{\frac{6}{5}}\left(\left[\frac{2 \pi}{\gamma}\right]+1\right)$ times the integral over $\mathbb{R}^{2}$.

Collecting the estimates and making use of (A.11), one obtains (A.7).

One more step is necessary to derive (A.4) from (A.7), because $x$ and $x^{\prime}$ are interior points. Let $x_{0}, x_{0}^{\prime} \in \partial \Lambda$ be the nearest points to $x, x^{\prime}$. The triangle inequality implies $l_{x}^{2}+\left|x_{0}-\xi\right|^{2} \leqq 9|x-\xi|^{2}$ for $\xi \in \partial \Lambda$, and the similar inequality for $x^{\prime}$. This reduces the integrals over $\xi, \tau$ in (A.5) to the form considered above, and the same majorization will apply.

If $l_{x^{\prime}} \geqq R / 4$, then:

$$
\begin{aligned}
\left|\frac{\partial}{\partial n_{\eta}} G^{0}\left(\sigma, \eta ; 0, x^{\prime}\right)\right| & \leqq \frac{8}{R} \cdot 2 \frac{\left|\eta-x^{\prime}\right|^{2}}{\sigma} G^{0}\left(\sigma, \eta ; 0, x^{\prime}\right) \\
& \leqq \frac{16 C_{0}}{\Gamma\left(\frac{1}{2}\right)} \sigma^{-3 / 2} \exp \left[-\frac{h l_{x^{\prime}}^{2}}{18 \sigma}\right] \exp \left[-h \frac{\left|\eta-x_{0}^{\prime}\right|^{2}}{2 \sigma}\right]
\end{aligned}
$$

and the integral over $\eta, \sigma$ can be majorized as above. If $l_{x^{\prime}}<R / 4$, the integration over $\eta \in \partial \Lambda$ will be divided into two parts. An integral over $S_{1}=\left\{\eta \in \partial \Lambda \mid \cos \left(\overrightarrow{n_{\eta}}, \overrightarrow{\eta x^{\prime}}\right) \geqq \frac{4\left|\eta-x^{\prime}\right|}{R}\right\}$ and one over $S_{2}=\partial \Lambda \backslash S_{1}$. The integral over $S_{2}$ can be majorized making use of (A.12). The integral over $S_{1}$ is treated by projecting on the tangent plane in $x_{0}^{\prime}$, extending the integral to $\mathbb{R}^{2}$ and explicitely performing the integrations. Note that in this last step the use of (A.9) is a too rough approximation.

Proposition A 3. For $t_{0}>0$, there is a constant $M\left(t_{0}\right)>0$ depending only on $t_{0}$, such that, for all $t \geqq t_{0}$ and all $\Lambda$ satisfying the condition of Proposition A2:

$$
\left|Z_{N}\left(t, x ; 0, x^{\prime}\right)\right| \leqq M\left(t_{0}\right) .
$$

Proof. From (A.4), it results that, for $t=t_{0}$ :

$$
G_{N}\left(t_{0}, x ; 0, x^{\prime}\right) \leqq\left(2 \pi t_{0}\right)^{-3 / 2}+C e^{\lambda t_{0}} t_{0}^{-3 / 2} .
$$


We shall prove, using a known argument [11], that

$$
\sup _{x, x^{\prime} \in \Lambda} G_{N}\left(t, x ; 0, x^{\prime}\right) \text { is a decreasing function of } t \text {. }
$$

Indeed:

$$
\begin{aligned}
& \frac{\partial}{\partial t} \int_{A}\left[G_{N}\left(t, x ; 0, x^{\prime}\right)\right]^{2 k} d x \\
& \quad=k \int_{\Lambda}\left[G_{N}\left(t, x ; 0, x^{\prime}\right)\right]^{2 k-1} \Delta_{x} G_{N}\left(t, x ; 0, x^{\prime}\right) d x \\
& \quad=-k(2 k-1) \int_{A}\left[G_{N}\left(t, x ; 0, x^{\prime}\right)\right]^{2 k-2}\left[\operatorname{grad}_{x} G_{N}\left(t, x ; 0, x^{\prime}\right)\right]^{2} d x
\end{aligned}
$$

therefore:

$$
\frac{\partial}{\partial t}\left[\int_{\Lambda}\left(G_{N}\left(t, x ; 0, x^{\prime}\right)\right)^{2 k} d x\right]^{1 / 2 k} \leqq 0
$$

and (A.15) follows by letting $k \rightarrow \infty$. From (A.14) and (A.15) one obtains (A.13) with:

$$
M\left(t_{0}\right)=2\left(2 \pi t_{0}\right)^{3 / 2}+C e^{\lambda t_{0}} t_{0}^{-3 / 2} .
$$

Acknowledgements. It is a pleasure for us to thank Professor C. Foias for his interest in this work, and to Drs. M. Bundaru and V. Protopopescu for many useful discussions.

\section{References}

1. Ruelle, D.: Helv. Phys. Acta 36, 789 (1963).

2. Robinson,D.W.: The thermodynamic pressure in quantum statistical mechanics. Lecture Note in Physics, Vol. 9. Berlin-Heidelberg- New York: Springer 1971.

3. Ginibre, J.: J. Math. Phys. 6, 238, 1432 (1965).

4. Novikov, I. D.: Funct. Anal. Appl. 3, 71 (1969) (Russian).

5. Nelson, E.: J. Math. Phys. 5, 332 (1964).

6. Ito, S.: Japan. J. Math. 27, 55 (1957).

Sato, K., Ueno, T.: J. Math. Kyoto Univ. 4, 529 (1965).

7. Arima, R.: J. Math. Kyoto Univ. 4, 207 (1964).

8. Rudin, W.: Real and complex analysis. New York: Mc Graw-Hill 1970.

9. Ginibre, J.: Some applications of functional integration in statistical mechanics. Lectures delivered at les Houches, 1970.

10. Friedman, A.: Partial differential equations of parabolic type. Englewood Cliffs, N.J.: Prentice-Hall Inc. 1964.

11. Guschin, A. K.: Sibir. Mat. J. 10, 43 (1969) (Russian).

\author{
N. Angelescu \\ G. Nenciu \\ Institute for Atomic Physics \\ P.O. Box 35 \\ Bucharest, Romania
}

